\title{
DISEÑO SÍSMICO DE ESTRUCTURAS DE ACERO BASADO EN CONFIABILIDAD ESTRUCTURAL Y CONCEPTOS DE ENERGÍA
}

\author{
Edén Bojórquez Mora ${ }^{(1)}$
}

\begin{abstract}
RESUMEN
Se propone un procedimiento para el diseño sísmico de edificaciones de acero que toma en cuenta la confiabilidad estructural. El procedimiento considera de manera explícita tanto las demandas máximas como las demandas por deformaciones plásticas acumuladas inducidas por sismos de larga duración en estructuras sismo-resistentes. El criterio de diseño se basa en suministrarle a las estructuras una capacidad para controlar las demandas máximas de ductilidad global, distorsión de entrepiso y de energía histerética disipada, mediante el uso de: a) espectros de ductilidad y espectros de energía histerética normalizada con tasas anuales de falla uniforme; y b) factores de transformación que toman en cuenta las diferencias entre las respuestas de sistemas de un grado y múltiples grados de libertad. Finalmente se ilustra el uso del procedimiento, que es aplicable a marcos estructurales de acero regulares diseñados de acuerdo a conceptos de capacidad, a través del diseño sísmico de un marco estructural de acero de ocho niveles y tres crujías.
\end{abstract}

\begin{abstract}
A procedure for the seismic design of steel buildings that takes into account structural reliability is proposed. The reliability-based procedure takes into account explicitly the maximum and cumulative plastic deformation demands induced by long duration ground motions in earthquakeresistant structures. The methodology is based in providing to the structures the capability to control the maximum demands of global ductility, interstory drift and dissipated hysteretic energy, through the use of: a) ductility and normalized hysteretic energy spectra with uniform annual failure rates; and b) transformation factors that take into account the differences between the response of single and multi-degree-of-freedom systems. Finally, the use of the procedure, which is applicable to regular steel frames that are designed according to the concepts of capacity design, is illustrated through its application to the seismic design of an eight-story three-bay structural steel frame.
\end{abstract}

\section{INTRODUCCIÓN}

Eventos sísmicos registrados en los últimos años, como los de México 1985, Northridge 1994, Kobe 1995 y Taiwán 1999, entre otros, han dejado de manifiesto la necesidad de mejorar las metodologías de diseño sísmico de estructuras, ya que una cantidad importante de estructuras diseñadas conforme a códigos sísmicos modernos no exhibieron un comportamiento adecuado durante dichos eventos. Por este

Artículo ganador de sexto concurso nacional de tesis de doctorado, recibido el 15 de diciembre de 2009 y aprobado para su publicación el 23 de diciembre de 2009. Se aceptarán comentarios y/o discusiones hasta cinco meses después de su publicación

(1) Facultad de Ingeniería, Universidad Autónoma de Sinaloa, Calzada de las Américas y Boulevard Universitarios $S / N$, Ciudad Universitaria, Culiacán, Sinaloa, México, C.P.80040.ebojorq@uas.uasnet.mx eden_bmseg@hotmail.com 
motivo, la comunidad internacional de ingenieros estructurales está actualmente intentando mejorar el diseño sísmico de edificios mediante la formulación de metodologías de diseño, cuyo objetivo es el control del daño estructural a través del control de los desplazamientos (Moehle 1992, Priestley 2000). Esto se puede ilustrar de las recomendaciones y conclusiones derivadas del Simposio Internacional sobre Metodologías de Diseño Sísmico para la Siguiente Generación de Códigos (Fajfar y Krawinkler 1997).

Actualmente, un gran porcentaje de códigos de diseño sísmico se basan en el uso de espectros de pseudoaceleración para estimar los requerimientos de resistencia y rigidez lateral de diseño de estructuras sismo-resistentes para controlar la demanda de desplazamiento lateral máximo. Sin embargo, en algunos casos otros parámetros podrían ser relevantes para el desempeño sísmico. En particular, la evidencia de campo y experimental sugieren que las demandas de deformación plástica acumulada pueden ser de relativa importancia en la seguridad estructural de edificios sismo-resistentes (Park y Ang 1985, Williams y Sexsmith 1997, Stephens y Yao 1987, Silva-Oliveria y López-Bátiz 2001). Las conclusiones obtenidas de investigación analítica y experimental, junto con la evidencia obtenida en campo después del terremoto de México en 1985, muestran que las estructuras sujetas a movimientos de banda angosta generados en la zona del lago de la Ciudad de México alcanzan severas demandas plásticas acumuladas, las cuales al no ser consideradas en el diseño pueden dar lugar a estructuras con un desempeño poco confiable (TeránGilmore 1996, Rodríguez y Aristizabal 1999, Huerta y Reinoso 2002, Bojórquez y Ruiz 2004, Arroyo y Ordaz 2007). Terán y Jirsa (2005) observaron que la energía histerética disipada demandada por movimientos de banda angosta puede ser tres o cuatro veces mayor a las de suelo firme. Por está razón, ellos obtuvieron la siguiente conclusión: "las metodologías de diseño sísmico basadas en el control de desplazamiento podrían proveer un adecuado nivel de seguridad para el diseño de estructuras con comportamiento histerético estable y sujetas a movimientos de suelo firme. Sin embargo, el uso de modelos de fatiga de bajo ciclaje debería ser considerado para el diseño de estructuras que exhiben deterioro rápido y excesivo del ciclo de histéresis, y para cualquier tipo de estructura sujeta a movimientos sísmicos de banda angosta y larga duración". Varios estudios similares ayudan a entender el porqué se obtuvo la siguiente conclusión durante el Simposio Internacional sobre Metodologías de Diseño Sísmico para la Siguiente Generación de Códigos (Fajfar y Krawinkler 1997): "El daño acumulado (energía disipada) debería ser considerado en el diseño de estructuras que sufren rápido deterioro de sus elementos, o estructuras sujetas a movimientos de larga duración".

Una forma de considerar el daño acumulado de manera explícita es a través de conceptos de energía sísmica. El uso de la energía sísmica fue inicialmente propuesto por Housner (1956) y ha sido utilizada por otros investigadores para fines de diseño sísmico (Akiyama 1985). Los métodos basados en conceptos de energía consisten en suministrar a la estructura una capacidad de energía superior o igual a la energía que le demandan los sismos (Uang y Bertero 1990). Se han desarrollado metodologías de diseño sísmico que se basan exclusivamente en el uso de la energía plástica histerética (Akbas et al. 2001, Choi y Kim 2006); sin embargo, dicho parámetro por sí solo puede resultar insuficiente al no considerar la historia completa del número y de la magnitud de los ciclos de comportamiento plástico. El control de la energía histerética, en complemento con el control de las demandas máximas (ductilidades y distorsiones máximas de entrepiso), puede ser una buena alternativa para garantizar un desempeño satisfactorio de las estructuras sismo-resistentes, sobre todo en las que presentan baja capacidad de acumulación de daño o que están sujetas a demandas sísmicas severas.

Otra limitación de la mayoría de los códigos de diseño sísmico en el mundo es no incluir los niveles de confiabilidad implícitos en las estructuras diseñadas con sus especificaciones. La mayoría de los reglamentos del mundo se basan principalmente en estudios de sistemas de un grado de libertad (S1GL) con comportamiento elasto-plástico perfecto para diseño sísmico de estructuras. Estos no garantizan una misma tasa de excedencia para la respuesta de S1GL y estructuras reales, como se ha observado en estudios recientes (Inoue y Cornell 1991, Esteva et al. 2005, Bojórquez et al. 2005). Además, los 
espectros de diseño sísmico de estructuras recomendados por dichos reglamentos en general no están asociados a niveles de confiabilidad o tasas anuales de falla (Cornell 1992, Wen 1995, Ghosh y Collins 2002, Rivera y Ruiz 2007).

En resumen, algunas limitaciones importantes en el diseño sísmico son la no consideración explicita del daño acumulado, la no inclusión de niveles prescritos de confiabilidad de las estructuras, y una estimación de la demanda máxima sísmica que se basa en estudios de S1GL. Por esta razón, el objetivo de este trabajo es introducir un procedimiento de diseño sísmico de estructuras de acero que considera de manera explícita el daño acumulado mediante la energía histerética normalizada respecto a la fuerza y desplazamiento de fluencia, así como la confiabilidad estructural.

\section{DEMANDA DE ENERGÍA HISTERÉTICA NORMALIZADA}

La energía histerética puede definirse a partir de la ecuación de movimiento de un S1GL:

$m \ddot{x}(t)+c \dot{x}(t)+f_{s}(x, \dot{x})=-m \ddot{x}_{g}(t)$

donde $m$ representa la masa del sistema, $c$ es el coeficiente de amortiguamiento viscoso del sistema, $f_{s}(x, \dot{x})$ es la fuerza restitutiva, $\ddot{x}_{g}$ es la aceleración del terreno, y $x$ es el desplazamiento relativo del sistema con respecto al del terreno; el punto sobre la literal indica derivada con respecto al tiempo. En el caso de un sistema elástico lineal, $f_{s}(x, \dot{x})=k x$, donde $k$ es la rigidez del sistema. Integrando cada miembro de la de la ecuación 1 con respecto a $x$ se tiene:

$$
\int m \ddot{x}(t) d x+\int c \dot{x}(t) d x+\int f_{s}(x, \dot{x}) d x=-\int m \ddot{x}_{g}(t) d x
$$

La ecuación 2 puede escribirse de la siguiente forma:

$$
E_{K}+E_{D}+E_{S}+E_{H}=E_{I}
$$

que representa el balance de energía en una estructura (Uang y Bertero 1990). En la ecuación $3, E_{K}, E_{D}$, $E_{S}$ y $E_{H}$ representan la energía cinética, energía de amortiguamiento, energía de deformación y la energía histerética disipada, respectivamente; y $E_{I}$ es la energía de entrada relativa. El término directamente relacionado con las demandas de deformación plástica acumulada es $E_{H}$.

De todos los tipos de energía absorbidos y disipados por una estructura, $E_{H}$ es la que está más relacionada con el daño estructural. La energía histerética disipada es igual al área total encerrada por cada uno de los ciclos de histéresis que desarrolla la estructura durante una excitación sísmica. Sin embargo, aunque $E_{H}$ proporciona información acerca de las demandas de deformación plástica acumuladas, una idea del número y magnitud promedio de los ciclos en los que una estructura incursiona en su comportamiento plástico, y puede resultar un parámetro apropiado para medir el daño estructural, su desventaja es que no aporta información suficiente acerca de la importancia que las demandas acumuladas de deformación plástica tienen en el desempeño sísmico de las estructuras, ya que se pueden tener valores similares de energía histerética en un sistema que tenga gran resistencia y desarrolle pocos ciclos de carga, y en un sistema con poca resistencia y que desarrolle muchos ciclos de carga (Terán-Gilmore 2001), y el daño que se presenta en ambos sistemas es completamente diferente. Un parámetro que se correlaciona 
mejor con el daño estructural es la energía histerética normalizada con respecto a la fuerza $F_{y}$ y desplazamiento de fluencia $\delta_{y}$ (Krawinkler y Nassar, 1992; Terán-Gilmore y Simon, 2006):

$$
E_{N}=\frac{E_{H}}{F_{y} \delta_{y}}
$$

En el presente trabajo se considera la energía histerética normalizada como parámetro para controlar el daño acumulado, a través de espectros de $E_{N}$ con tasa anual de falla uniforme (TAFU), los cuales se definen en la siguiente sección.

\section{Espectros de energía histerética normalizada con tasa anual de falla uniforme}

Las incertidumbres asociadas a la respuesta de estructuras sujetas a la acción de los sismos, hacen que el problema sísmico se defina en términos probabilísticos. Por tal motivo, uno de lo objetivos fundamentales de la ingeniería sísmica es cuantificar que tan confiable es una estructura considerando todo el posible peligro sísmico al cual será sometida durante su vida útil. Una herramienta para estimar la confiabilidad de estructuras sujetas a acciones sísmicas es través de la evaluación de la tasa media anual de excedencia (TAE) de un parámetro asociado a la demanda sísmica $(D S)$, por ejemplo: la distorsión máxima de entrepiso, la energía histerética disipada, el índice de daño de Park y Ang, etc. Mediante estudios pasados desarrollados por Esteva (1967) y Cornell (1968) y el uso del teorema de la probabilidad total, la TAE se puede obtener mediante la siguiente expresión:

$v_{D S}(x)=\sum_{i} v_{i} \iint_{I S} \int_{M} P[D S>x \mid I S, M, R] f(I S \mid M, R) f(M, R) d r d m d(i s)$

donde $v_{D S}(x)$ es la tasa media anual de que $D S$ exceda un valor $x, v_{i}$ es la tasa de sismos para una fuente específica que afecta el sitio de interés, $f(I S \mid M, R)$ es la función de distribución condicional de la medida de intensidad sísmica $(I S)$ utilizada dados la magnitud $(M)$ y la distancia $(R)$ (ley de atenuación sísmica). $f(M, R)$ es la función de distribución de probabilidad conjunta de $M$ y $R$. Finalmente, $P[D S>x \mid I S, M, R]$ es la probabilidad de excedencia de $D S$ dados $I S, \quad M \quad$ y $R$. Si $P[D S>x \mid I S, M, R]=P[D S>x \mid I S]$ se dice que la medida de $I S$ es suficiente (Bazzurro 1998, Shome 1999) debido a que la predicción de la respuesta estructural es independiente de $M$ y $R$ cuando se utiliza cierta $I S$. Se ha demostrado que la pseudoaceleración espectral en el modo fundamental de vibración de una estructura $S a\left(T_{1}\right)$ es suficiente con respecto a la magnitud y a la distancia (Shome 1999). Sin embargo, es importante enfatizar que bajo algunas circunstancias $S a\left(T_{1}\right)$ no resulta un buen predictor de la respuesta estructural no-lineal, y por lo tanto se requieren medidas de intensidad sísmica más apropiadas (especialmente aquellas con una íntima relación con la forma espectral). Por ejemplo, el vector $\langle S a, \varepsilon\rangle$, el cual se relaciona con la forma espectral elástica (Baker y Cornell 2005). Otras medidas incluyen el escalar propuesto por Tothong y Luco (2007); el vector $<S a, R_{T 1, T 2}>$ propuesto por Baker y Cornell (2008); y el vector $\left\langle S a, N_{p}\right\rangle$ propuesto por Bojórquez y Iervolino (2009). En este trabajo los registros sísmicos se seleccionaron de tal manera que el escalamiento basado en $\mathrm{Sa}\left(T_{1}\right)$ resulta adecuado debido a: i) la suficiencia de $S a\left(T_{1}\right)$ con respecto a $M$ y $R$; y ii) la similitud en la forma espectral de los registros sísmicos. Por este motivo, la ecuación 5 puede expresarse de la siguiente manera: 
$v_{D S}(x)=\int_{S a\left(T_{1}\right)} P\left[D S>x \mid S a\left(T_{1}\right)=s a\right] d v_{S a\left(T_{1}\right)}(s a)$

donde $d v_{S a\left(T_{1}\right)}(s a)=v_{S a\left(T_{1}\right)}(s a)-v_{S a\left(T_{1}\right)}(s a+d s a)$ es el diferencial de la curva de peligro sísmico expresada en términos de $S a\left(T_{1}\right)$. ecuación:

De la misma forma, la tasa media anual de falla en una estructura se evalúa con la siguiente

$$
v_{F}=\int_{S a\left(T_{1}\right)} P\left[Q \geq 1 \mid S a\left(T_{1}\right)=s a\right] d v_{S a\left(T_{1}\right)}(s a)
$$

donde $Q$ se expresa como la relación entre la demanda y la capacidad. Para el caso de la energía histerética normalizada disipada $Q=\frac{E_{N D}}{E_{N C}}$; es decir, es la relación entre la demanda de energía histerética normalizada y la capacidad. $P\left[Q \geq 1 \mid S a\left(T_{1}\right)=s a\right]$ es la probabilidad de que se presente la falla del sistema, dada una $\mathrm{Sa}\left(T_{1}\right)$.

La metodología para obtener los espectros con tasa anual de falla uniforme es la siguiente (Rivera 2006, Rivera y Ruiz 2007):

- Se establece el valor del coeficiente sísmico $\left(C_{y}\right)$ y periodo del sistema $(T o)$.

- Se escalan los registros para que en el periodo correspondiente To tengan la pseudoaceleración deseada (Shome y Cornell, 1999; Chan et al. 2005).

- Se somete un S1GL cuyas propiedades corresponden a $C_{y}$ y $T o$ a los registros escalados y se obtiene la demanda sísmica correspondiente a cada registro.

- Se evalúa la probabilidad de que la estructura falle $(Q \geq 1)$ para cierto nivel de intensidad sísmica (donde la intensidad está dada en términos de la pseudoaceleración) $P\left[Q \geq 1 \mid S a\left(T_{1}\right)=s a\right]$. Dicha probabilidad se determina como el número de registros que provocan la falla del sistema entre el número total de registros utilizados (distribución uniforme); sin embargo existen otras alternativas para determinar dicha probabilidad, como el caso de la regresión logística. $P\left[Q \geq 1 \mid S a\left(T_{1}\right)=s a\right]$ representa las curvas de vulnerabilidad sísmica del sistema.

- Con la probabilidad de falla y las curvas de peligro sísmico de un sitio de interés, se obtiene la tasa anual de falla del sistema $v_{F}$.

- Se repite el proceso para otras intensidades.

- Se generan las curvas de peligro de demanda estructural para diferentes periodos de vibración estructural. 
- Se selecciona el valor de la tasa anual de falla. Para dicho valor se lee el coeficiente sísmico asociado a un periodo. Finalmente se trazan los espectros con TAFU.

En la figura 1 se ilustra un ejemplo de un espectro de energía histerética normalizada asociado a una $\operatorname{TAFU}\left(\nu_{F O}\right)$.

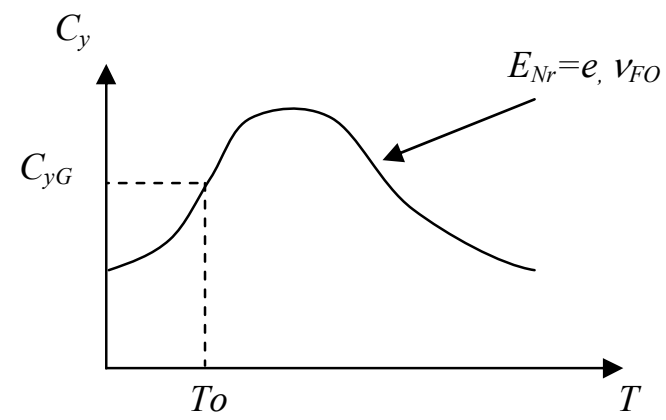

Figura 1. Espectro correspondiente a una tasa anual de falla $v_{F O}$ de un S1GL con comportamiento bilineal y una energía histerética normalizada requerida $E_{N r}=\mathrm{e}$.

\section{CAPACIDAD DE ENERGÍA HISTERÉTICA NORMALIZADA}

En este trabajo, la capacidad de disipación de energía histerética normalizada global en un marco estructural de acero se define mediante la siguiente expresión:

$E_{N C G}=\frac{E_{H C G}}{C_{y G} D_{y G} W}$

donde $E_{H C G}$ es la capacidad de disipación de energía histerética del sistema global, que se estima como se ilustrará mas adelante. $D_{y G}$ y $C_{y G}$ son el desplazamiento y el coeficiente sísmico global de fluencia que se obtienen mediante un análisis de empuje lateral estático no lineal, y $W$ es el peso total de la estructura.

Con el fin de evaluar la capacidad de disipación de energía histerética en un marco regular de acero se hacen las siguientes hipótesis: a) el comportamiento plástico debe concentrarse en las vigas que integran la estructura (la estructura debe diseñarse como un sistema viga débil-columna fuerte, para que las columnas no exhiban daño considerable) y b) el nivel de daño estructural en las vigas que integran un mismo piso debe ser similar.

Bojórquez et al. (2006) demostraron que para el caso de marcos estructurales de acero regulares el daño es bastante similar en las vigas que integran un mismo piso. En el caso de estructuras irregulares, el procedimiento puede usarse siempre y cuando se establezca la contribución de cada elemento en la capacidad total de disipación de energía de la estructura.

Con la hipótesis de que las vigas son los únicos elementos capaces de disipar energía por comportamiento no lineal, la capacidad de disipación de energía histerética de la estructura debería ser igual a la suma de la capacidad de energía histerética disipada por las vigas. Sin embargo, cuando una 
estructura se sujeta a la acción de un sismo, no todas las vigas desarrollan el total de su capacidad de disipación de energía histerética, por lo que es necesario establecer la contribución de cada viga a la capacidad total del sistema. Debido a que la segunda condición establece que las vigas que integran un mismo piso sufren un daño similar, es suficiente estimar la capacidad de energía histerética en la estructura a través del conocimiento de la disipación de dicha energía a lo alto de la estructura. En este trabajo, la distribución de la energía en la altura del edificio se realiza mediante un factor de participación de energía histerética $F_{E H i}$. Dicho factor indica el grado de participación de cada piso en la capacidad total de disipación de energía de un edificio. Esto implica que la capacidad de disipación de energía de cada piso se corrige por dicho factor conforme a lo que establece la ecuación 9, donde la capacidad de disipación de energía histerética de la estructura es igual a la suma de la capacidad de cada piso multiplicada por su correspondiente factor de participación $F_{E H i}$.

$$
E_{N C G}=\frac{\sum_{i=1}^{N_{P}} F_{E H i} E_{H C i}}{C_{y G} D_{y G} W}
$$

donde $N_{P}$ es el número de pisos en la estructura, $E_{H C i}$ es la capacidad de disipación de energía histerética del piso $i$, y $F_{E H i}$ es el factor de participación de energía histerética del piso $i$.

Para estimar la contribución de los distintos miembros estructurales a la capacidad de disipación de energía histerética de las edificaciones de acero, usualmente se supone una distribución de energía histerética a lo largo de la altura. Mientras que Akbas et al. (2001) propusieron una distribución lineal, estudios recientes sugieren que, si la disipación de energía se concentra en las vigas de un marco estructural, una distribución lognormal es más representativa de la manera en que se distribuye la energía plástica a lo largo de la altura (Bojórquez 2007). Un factor de participación de energía histerética $F_{E H}$ puede establecerse para estimar la contribución de cada piso a la capacidad total de la edificación. En particular, $F_{E H}$ puede formularse de tal manera que se evalúe el porcentaje de capacidad de energía última que un piso disipa durante un movimiento sísmico (los pisos críticos contribuirán con su capacidad total; lo cual se expresa mediante un valor unitario para $F_{E H}$ ). El parámetro $F_{E H}$ para un piso en particular se estima como la relación entre la energía histerética disipada por dicho piso dividida por la energía histerética disipada en el piso crítico; por está razón $F_{E H}$ para el piso crítico tiene un valor unitario. Bojórquez et al. (2008) mediante el análisis de ocho marcos estructurales de acero sometidos a 31 movimientos de larga duración generados en el suelo blando del valle de México, obtuvieron la siguiente expresión para estimar $F_{E H}$ en marcos estructurales de acero:

$$
F_{E H}=\min \left(F_{E H}^{*}, 1\right)
$$

donde:

$$
F_{E H} *=\frac{1}{(-0.0675 \mu+2.82) h / H} \exp \left\{-\frac{1}{2}\left[\frac{(\ln (h / H)-\ln (0.031 \mu+0.3461))}{0.06 \mu+0.39}\right]^{2}\right\}
$$

$h / H$ representa la altura de un nivel normalizada por la altura total de la estructura $(\mathrm{H})$ relativa al nivel del suelo, y la $\mu$ ductilidad desarrollada por el sistema. 
La figura 2 muestra la evolución del valor de $F_{E H}$ a lo largo de la altura del edificio al incrementarse la ductilidad. Se observan incrementos en los valores de $F_{E H}$, en particular en los pisos más altos a medida que se incrementa la ductilidad. Esto indica que las vigas localizadas en los pisos más altos tienden a contribuir más a la capacidad de disipación de energía de la estructura, a medida que se incrementa la ductilidad global desarrollada por una estructura.

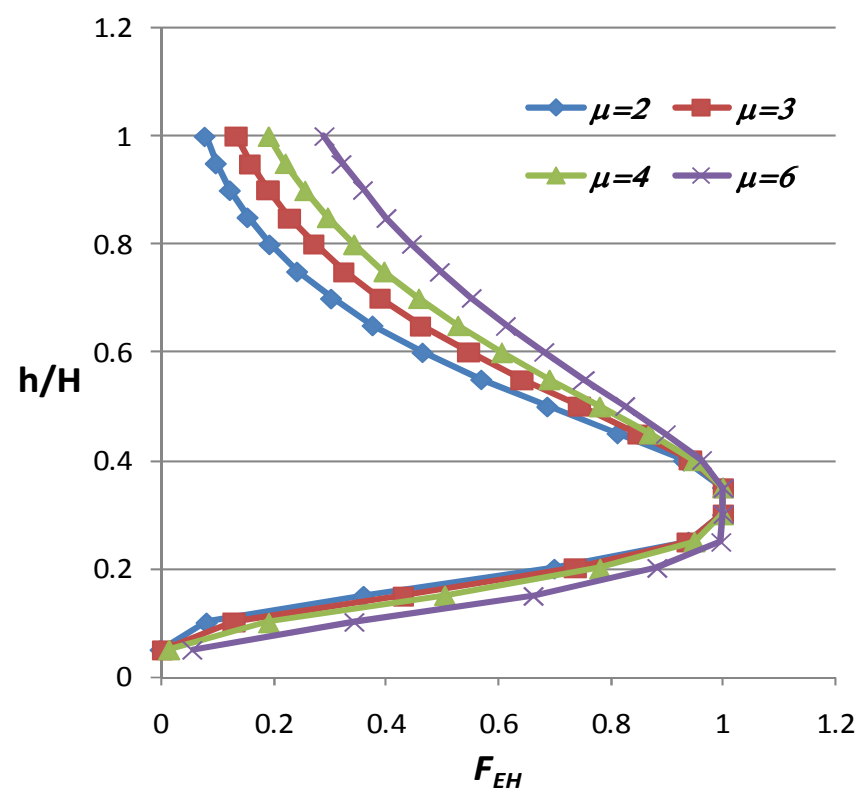

Figura 2. Evolución de $F_{E H}$ al incrementarse la ductilidad global.

Aunque la manera anterior de establecer $F_{E H}$ tiene la limitación de no considerar la capacidad de energía de miembros individuales, la cual podría variar de piso a piso y de crujía a crujía, y podría resultar necesario obtener una distribución del daño a lo largo de la altura en la estructura, en lugar de la configuración de energía histerética disipada. Bojórquez et al. (2009) determinaron que $F_{E H}$ obtenido de esta manera provee de una aproximación razonable acerca de cómo los miembros estructurales contribuyen a la capacidad total de energía histerética de estructuras a base de marcos. Por lo tanto, recomiendan utilizar dicha expresión para estimar $F_{E H}$, y esa es la razón por la cual se utiliza en el presente trabajo.

Para evaluar la capacidad de disipación de energía histerética en un elemento estructural se consideró la ecuación propuesta de (Akbas et al. 2001) para el caso de elementos de acero con sección W, como se ilustra a continuación:

$E_{H e}=2 Z_{f} f_{y} \theta_{p a}$

donde $Z_{f}$ es el módulo de sección plástico de los patines, $f_{y}$ es el esfuerzo de fluencia, y $\theta_{p a}$ es la capacidad de rotación plástica acumulada del elemento. Esta ecuación considera que únicamente los patines del elemento disipan energía por deformación plástica. 
Sustituyendo la ecuación 11 en la 9 se obtiene la siguiente ecuación, que representa la capacidad de energía histerética normalizada correspondiente a marcos estructurales de acero:

$E_{N C G}=\frac{\sum_{i=1}^{N_{P}}\left(2 N_{C} Z_{f} F_{y} \theta_{p a} F_{E H i}\right)}{C_{y G} D_{y G} W}$

donde $N_{C}$ representa el número de crujías.

\section{DISEÑO SÍSMICO DE ESTRUCTURAS DE ACERO BASADO EN CONFIABILIDAD ESTRUCTURAL Y CONCEPTOS DE ENERGÍA}

Se propone un criterio de diseño sísmico de marcos estructurales de acero que se basa en el uso de S1GL equivalentes que representan las características de una estructura de acero de varios grados de libertad. Se utilizan espectros de energía histerética normalizada con TAFU. Al determinar los requerimientos en S1GL equivalentes, es posible mediante factores de transformación de respuesta determinar los requerimientos en marcos estructurales de acero reales (Bojórquez y Ruiz 2007). En este trabajo los sistemas equivalentes (que representan las características de las estructuras) se refieren a S1GL con coeficiente sísmico $\left(C_{y}\right)$, periodo fundamental de vibración $(T o)$ y porcentaje de amortiguamiento crítico $(\xi)$ iguales a los que están asociados al SMGL.

El criterio propuesto se basa en revisar tres condiciones de diseño: 1) el requerimiento de resistencia lateral (a través del coeficiente sísmico $C_{y}$ ), para no exceder el valor de la ductilidad global disponible, asociada con una tasa anual de falla $v_{F O I}$ seleccionada para este estado límite, 2) el control de la distorsión máxima de entrepiso asociada con una tasa anual de falla $v_{F O 2}$, y 3 ) el requerimiento de energía histerética disipada asociada a una tasa anual de falla $v_{F O 3}$. Por simplicidad aquí se supone $v_{F O I}=v_{F O 2=} v_{F O 3}=v_{F O}$.

\section{Criterio de diseño propuesto}

Una metodología de diseño sísmico requiere que la respuesta de una estructura se compare con la capacidad que tiene ésta para acomodarla en función del desempeño sísmico requerido. Metodologías de diseño propuestas recientemente contemplan esta revisión en tres distintas etapas:

a) Prediseño Global. Estimación de manera rápida y razonable de las demandas sísmicas globales y compararlas con los niveles de capacidad globales. Un uso adecuado de los espectros de respuesta proporciona información que permite determinar las características mecánicas globales (coeficiente sísmico, periodo de vibración, amortiguamiento y capacidad de deformación última) que requiere una estructura.

b) Diseño Local Preliminar. Una vez que se cuenta con las características globales en una estructura, es necesario establecer las propiedades estructurales y detallado a nivel local (obtención del tamaño, resistencia y detallados de los elementos estructurales).

c) Revisión del Diseño Preliminar. Se han propuesto algunas recomendaciones para la revisión del diseño preliminar a través de una serie de análisis dinámicos estructurales que permitan establecer el desempeño de las estructuras a nivel local y global. 
Varios autores han identificado la importancia del Prediseño Global y Diseño Local Preliminar, y varias metodologías se han propuesto para estos dos pasos del diseño (Bertero y Bertero 1992, Priestley 2000). Terán y Simon (2006) propusieron una metodología de prediseño para contemplar estos dos pasos del diseño en un formato que fomenta el control de las demandas máximas y acumuladas. Es importante mencionar que el uso del criterio propuesto se basa en el control de la falla, y que requiere de un prediseño ya establecido, el cual se puede realizar mediante las metodologías antes descritas, mediante algún código de diseño sísmico, o alternativamente el prediseño puede corresponder a un diseño ante cargas gravitatorias. Para lograr el objetivo aquí planteado, el criterio utiliza dos curvas de peligro de demanda estructural, dos diferentes tipos de espectros con TAFU (espectro de ductilidad y de energía histerética normalizada); y tres factores de transformación para considerar las diferencias entre S1GL y marcos estructurales de acero (uno para ductilidad, otro para distorsiones máximas de entrepiso y uno para energía histerética normalizada).

El criterio de diseño propuesto se ilustra en la figura 3 y se describe a continuación:

\section{Confiabilidad deseada en la estructura.}

La confiabilidad deseada en la estructura se determina aquí en términos de la tasa anual de falla estructural. El primer paso es establecer la capacidad de ductilidad global disponible $\left(\mu_{G}\right)$ de la estructura, y la distorsión máxima de entrepiso tolerable $\left(\gamma_{t o l}\right)$, ambos asociados a una tasa anual de falla $\left(v_{F O}\right)$. Estos valores deben ser proporcionados por los códigos sísmicos en el futuro o, alternativamente pueden evaluarse para una estructura en particular diseñada de acuerdo con algún código de diseño que contenga niveles de seguridad mínimos comúnmente aceptados por la sociedad.

\section{Resistencia lateral requerida para el control de la ductilidad máxima.}

Este paso consiste en determinar los requerimientos de resistencia lateral (coeficiente sísmico requerido $C_{y r e q}$ ) de la estructura a través de los espectros de ductilidad constante con TAFU (figura 4) en función del periodo de la estructura $T o$, la capacidad de ductilidad $\mu_{G}$ y la tasa anual de falla deseada en la estructura $v_{F O}$.

Debido a que los espectros se obtienen a partir de S1GL, se utiliza un factor de transformación de ductilidad $T_{\mu}$ (Bojórquez y Ruiz 2007) para considerar la diferencia que existe entre la respuesta de un marco estructural de acero y de un S1GL. Es conveniente mencionar que debido a que el S1GL equivalente que representa la estructura tiene el mismo periodo, coeficiente sísmico y amortiguamiento, los espectros de S1GL pueden utilizarse para determinar los requerimientos de resistencia en la estructura real por ser los mismos; sin embargo, la ductilidad desarrollada por la estructura y el sistema equivalente difieren, por lo que es necesario usar el factor de transformación de ductilidad.

En la figura 4 se ilustra un espectro de ductilidad $\mu_{l}$ (ductilidad en el S1GL) asociado a una TAFU, y la forma en que se obtiene el $C_{y r e q}$ para una estructura que tiene un periodo $T o$, y una capacidad de ductilidad de su sistema equivalente $\mu_{1}$ asociada a una tasa anual de falla $v_{F O}$. Los pasos detallados para calcular este tipo de espectros se pueden encontrar en Rivera (2006) y Rivera y Ruiz (2007). 

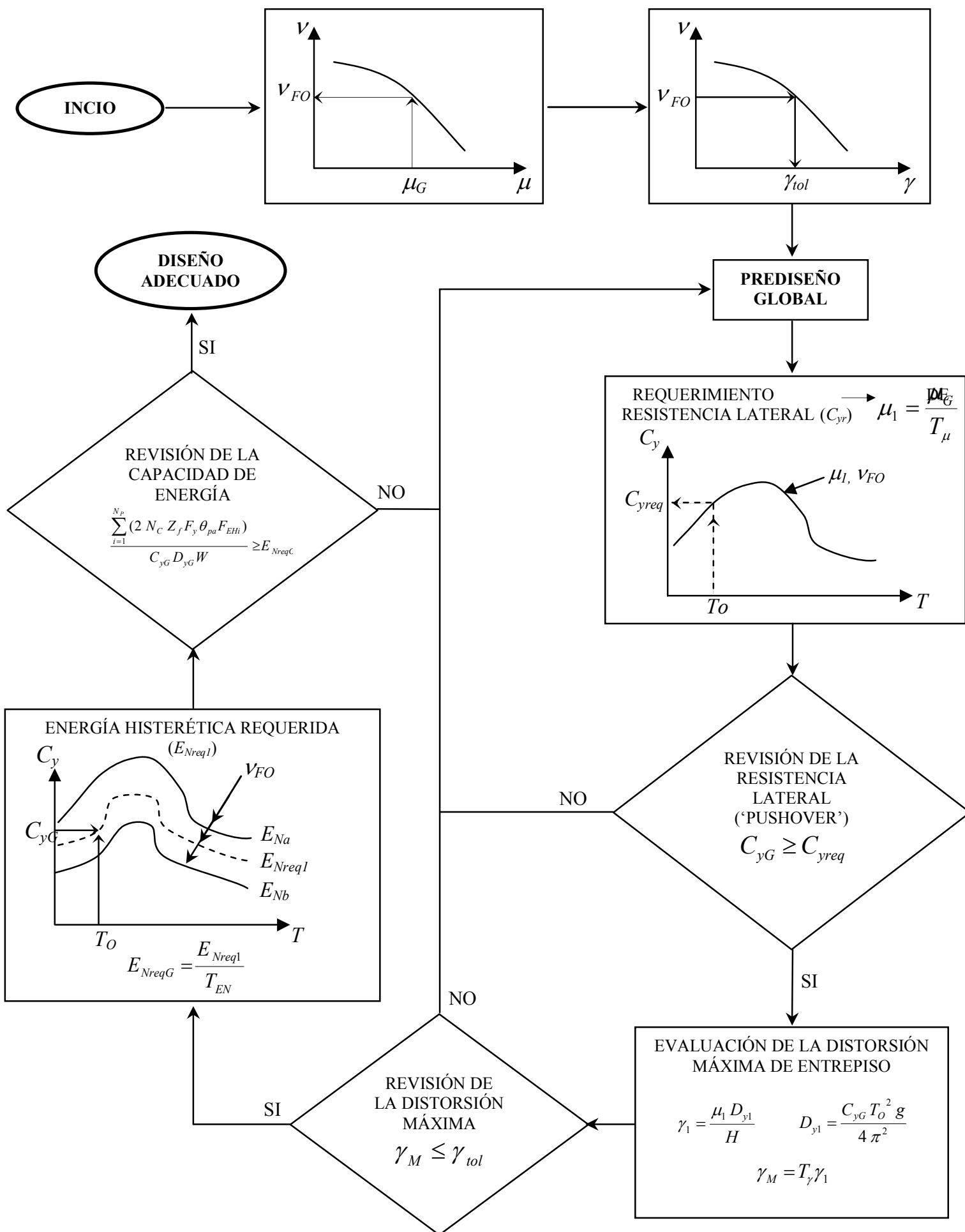

Figura 3. Diagrama de flujo del criterio propuesto. 


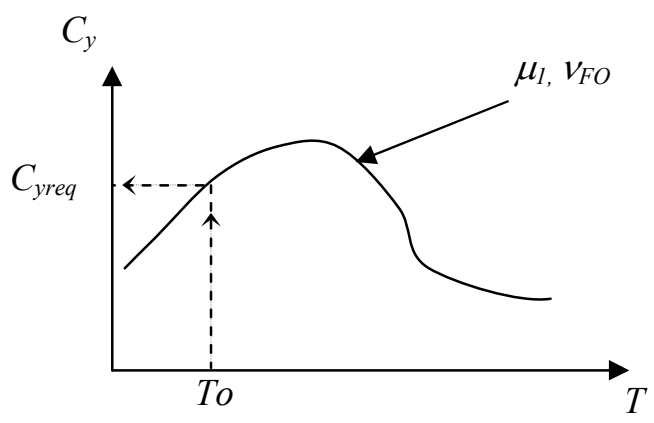

Figura 4. Espectro correspondiente a una tasa anual de falla $v_{F O}$ de un S1GL con comportamiento bilineal y ductilidad $\mu_{1}$.

\section{Revisión de la resistencia lateral requerida.}

La primera condición de diseño que consiste en garantizar la resistencia lateral requerida, se revisa mediante un análisis de empuje lateral estático no-lineal (figura 5) de la estructura. Con este análisis se evalúa el coeficiente sísmico real de la estructura y se compara con el coeficiente sísmico requerido, si $C_{y G}$ $\geq C_{y r e q}$, entonces se continúa con el procedimiento, de lo contrario la estructura debe rediseñarse.

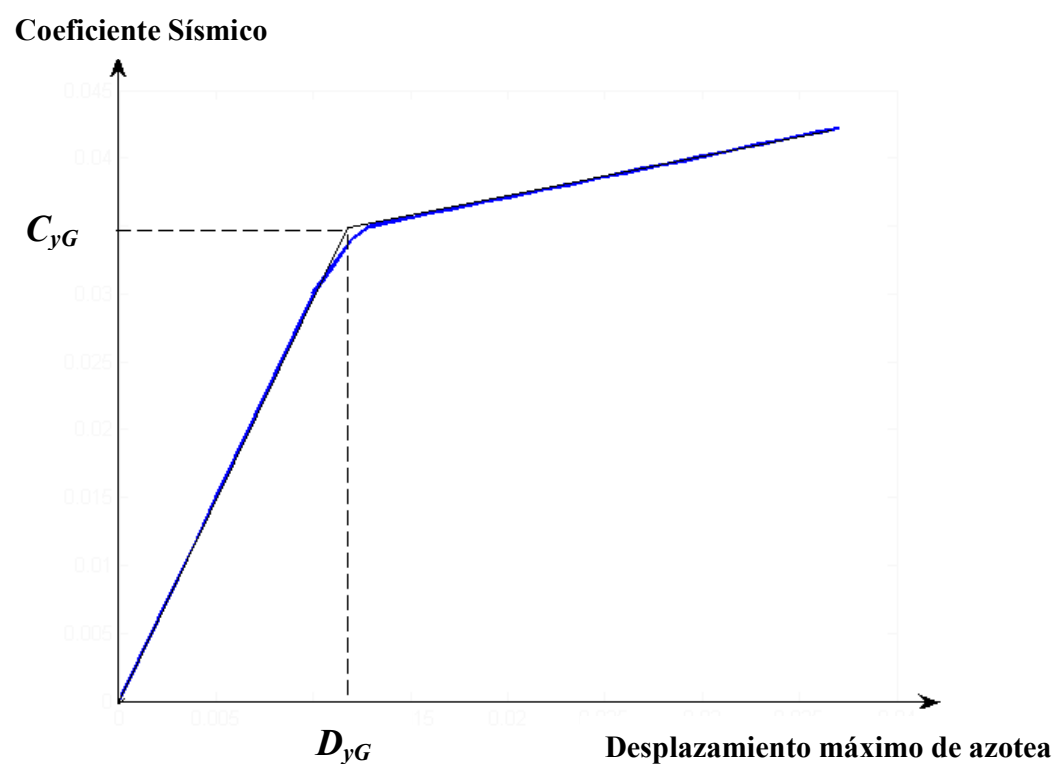

Figura 5. Ejemplo de un análisis de empuje lateral estático no-lineal ('push-over').

4. Revisión de la distorsión máxima de entrepiso.

La distorsión máxima de entrepiso en la estructura se determina a partir de la distorsión máxima en el S1GL. La distorsión máxima en el modelo simplificado se evalúa de la siguiente manera:

$\gamma_{1}=\frac{\mu_{1} D_{y 1}}{H}$ 
donde $\mu_{1}$ y $D_{y 1}$, son la demanda de ductilidad y el desplazamiento de fluencia en el S1GL, respectivamente, y $H$ la altura total. $D_{y 1}$ se evalúa con la siguiente expresión:

$D_{y 1}=\frac{C_{y_{\text {real }}} T_{o}^{2} g}{4 \pi^{2}}$

Enseguida, la distorsión máxima de entrepiso se determina como:

$\gamma_{M}=T_{\gamma} \gamma_{1}$

donde $T_{\gamma}$ es el factor de transformación de distorsión máxima de entrepiso (Bojórquez y Ruiz 2007), y $\gamma_{M}$ la demanda de distorsión máxima de entrepiso en la estructura.

Finalmente, se compara la distorsión máxima de entrepiso con la condición de diseño que en este caso es $\gamma_{t o l}$ (asociada a $\left.v_{F O}\right)$ :

$\gamma_{M} \leq \gamma_{t o l}$

Si la distorsión máxima de entrepiso $\gamma_{M}$ es menor o igual que la tolerable $\gamma_{t o l}$ (asociada a una $v_{F O}$ ), entonces se satisface la segunda condición de diseño, de lo contrario la estructura debe rediseñarse.

5. Energía histerética normalizada que debe ser capaz de soportar la estructura.

En esta etapa se evalúan las demandas acumuladas esperadas a través del espectro de energía histerética normalizada (figura 6). Para ello, en esta sección se introducen los espectros asociados a una tasa anual de falla $v_{F O}$. Con el periodo de la estructura $T o$ y el coeficiente sísmico real $C_{y G}$, se obtiene la energía histerética normalizada requerida en el S1GL que representa las características de la estructura $E_{\text {NreqI }}$, donde $E_{\text {NreqI }}=E_{H I} / F_{y} D_{y}$ representa la relación entre la energía histerética en el S1GL $\left(E_{H I}\right)$ dividida entre la fuerza y el desplazamiento de fluencia.

Una vez obtenida $E_{\text {Nreq } 1}$ el siguiente paso es obtener la energía histerética normalizada requerida en la estructura $E_{N r e q G}$, para ello se utiliza el factor de transformación de energía histerética $T_{E N}$ (Bojórquez y Ruiz 2007) que relaciona la energía entre el S1GL y el marco estructural.

$E_{N \text { reqG }}=\frac{E_{N \text { req } 1}}{T_{E N}}$

6. Revisión de la capacidad de disipación de energía.

Para satisfacer los requisitos de energía se requiere que se cumpla la siguiente condición:

$E_{\text {NCG }} \geq E_{\text {NreqG }}$

donde $E_{N C G}$ es la capacidad de energía histerética normalizada en la estructura. 
La capacidad de disipación de energía puede estimarse mediante la expresión 12, que sustituyendo en la ecuación 18 resulta:

$$
\frac{\sum_{i=1}^{N P}\left(2 N C Z_{f} F_{y} \theta_{p a} F p_{E H i}\right)}{C_{y G} D_{y G} W} \geq E_{N r e q G}
$$

Finalmente, si se cumple la ecuación 19 el diseño propuesto es adecuado, de lo contrario debe rediseñarse la estructura.

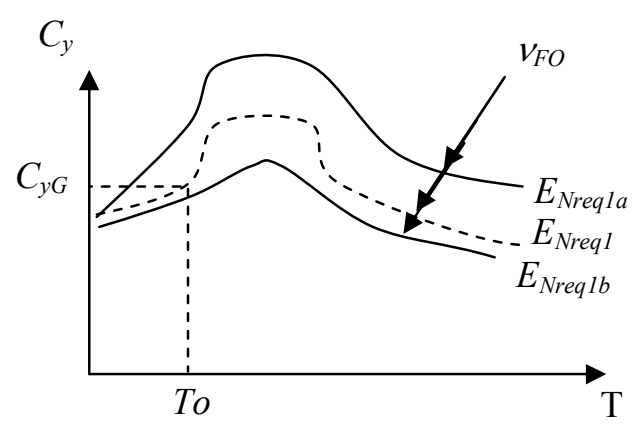

Figura 6. Espectros de energía histerética normalizada asociados a una tasa anual de falla $v_{F O}$.

\section{EJEMPLO DE APLICACIÓN}

El criterio de diseño propuesto se aplica a un marco estructural de acero de ocho niveles y tres crujías que se considera ubicado en la zona IIIb del valle de México. Las características geométricas del marco se muestran en la figura 7. Es importante mencionar que se parte de un prediseño que puede ser establecido mediante un código de diseño sísmico o algún procedimiento simplificado. Se utilizaron secciones W de acero (AISC, 2003) tanto para las vigas como para las columnas. En la tabla 1 se muestran las secciones $\mathrm{W}$ en los diferentes niveles del marco estructural.

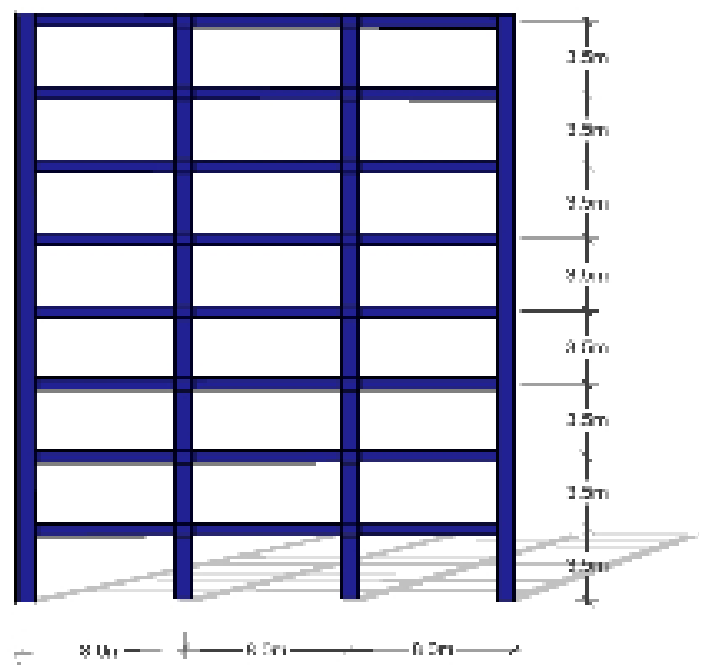

Figura 7. Geometría del marco estructural. 
Tabla 1. Secciones propuestas para la estructura.

\begin{tabular}{|c|c|c|c|}
\hline Nivel & Vigas & $\begin{array}{c}\text { Columnas } \\
\text { Internas }\end{array}$ & $\begin{array}{c}\text { Columnas } \\
\text { Externas }\end{array}$ \\
\hline 1 & W24x76 & W36x194 & W36x194 \\
\hline 2 & W24x94 & W36x194 & W36x194 \\
\hline 3 & W24x94 & W36x170 & W36x 170 \\
\hline 4 & W24x94 & W36x170 & W36x 170 \\
\hline 5 & W24x76 & W36x150 & W36x150 \\
\hline 6 & W21x62 & $\mathrm{W} 36 \times 150$ & W36x 150 \\
\hline 7 & $\mathrm{~W} 21 \times 50$ & W36x135 & W36x135 \\
\hline 8 & $\mathrm{~W} 21 \times 50$ & W36x135 & W36x 135 \\
\hline
\end{tabular}

\section{Confiabilidad deseada en la estructura}

El primer paso para el diseño sísmico consiste en establecer el nivel de confiabilidad del sistema a través de la tasa anual de falla estructural, en este caso se estable una tasa anual de falla $v_{F O}=0.003$ que está asociada a una capacidad de ductilidad global de la estructura $\mu_{G}=3$. La tasa anual de falla se seleccionó con base en las curvas de peligro de demanda que presentaría un marco estructural de acero diseñado con el RCDF-2004 con características similares a la estructura que se diseñará con el método propuesto. La figura 8 ilustra la correspondiente curva de peligro de demanda para una estructura con características similares e igual número de niveles a la considerada en el ejemplo ilustrativo. Se observa que la tasa anual de falla asociada a $\mu_{G}=3$ es igual a 0.003 . Se supone que tanto la ductilidad, la distorsión máxima de entrepiso y la energía histerética normalizada estarán asociadas a un mismo nivel de tasa anual de falla. La distorsión máxima de entrepiso resulta $\gamma_{t o}=0.023$ para una $v_{F O}=0.003$ de acuerdo a la curva de peligro establecida por Bojórquez et al. (2007) para la distorsión (ver figura 9). Finalmente, la energía histerética normalizada con respecto a la fuerza y resistencia de fluencia del sistema $\left(E_{N G}\right)$ asociada a $v_{F O}=0.003$ se obtendrá más adelante (mediante un espectro de $E_{N G}$ con TAFU).

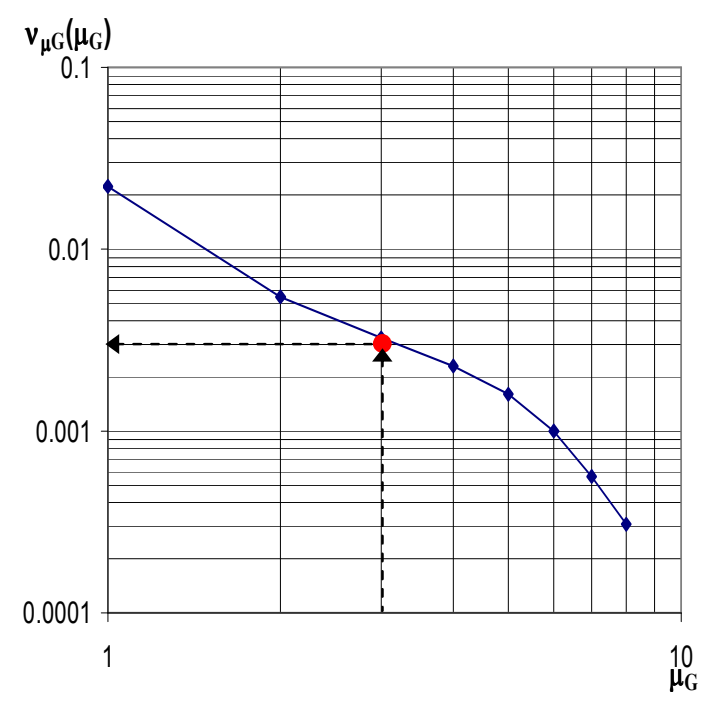

Figura 8. Curva de peligro de ductilidad para un marco de acero de ocho niveles. 


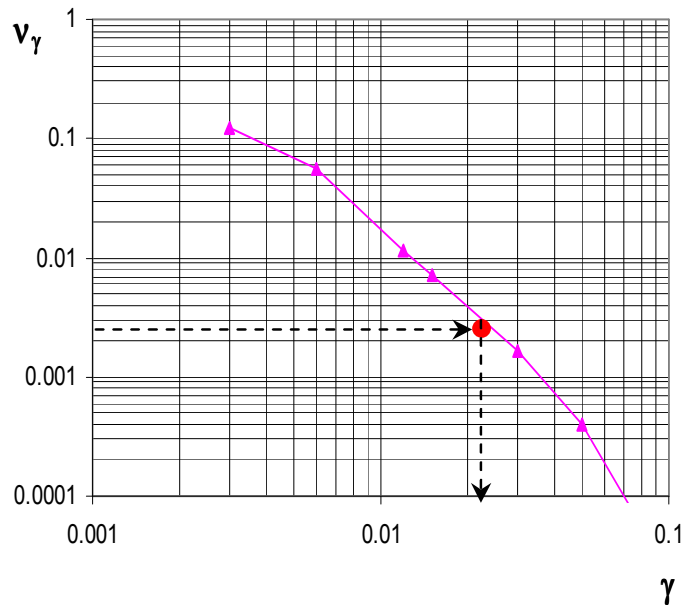

Figura 9. Curva de peligro de distorsión máxima de entrepiso para un marco de acero de ocho niveles.

\section{Resistencia lateral requerida para el control de la ductilidad máxima}

El coeficiente sísmico requerido se determina utilizando el espectro de ductilidad para $v_{F O}=0.003$. Los espectros utilizados en este trabajo (tanto de ductilidad como de energía histerética asociados a una TAFU) se obtuvieron a partir de 31 acelerogramas reales registrados en la zona IIIb del valle de México. La figura 10 ilustra los espectros de respuesta elásticos para $3 \%$ de amortiguamiento de los 31 acelerogramas utilizados en este estudio. Mientras que este nivel de amortiguamiento ha sido utilizado en otros estudios para estructuras de acero (Popov et al. 1989), los registros están escalados para la pseudoaceleración en el periodo de vibración de la estructura $(T o=1.07 \mathrm{~s})$.

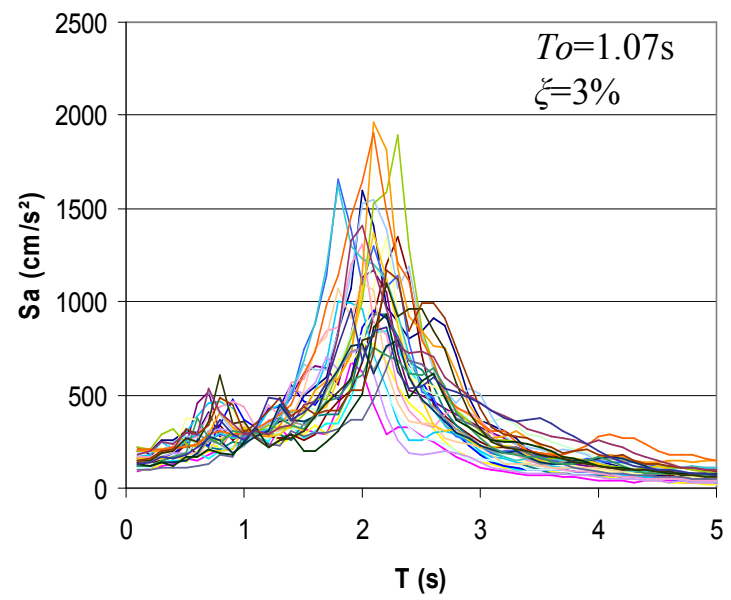

Figura 10. Espectros de respuesta elásticos de los registros sísmicos utilizados para la obtención de los espectros con tasa anual de falla uniforme.

Se deben tomar en cuenta las diferencias entre la respuesta de una estructura y su S1GL equivalente, ya que dichos espectros se obtuvieron para S1GL. Esto se realiza mediante el factor de transformación de ductilidad. Utilizando el factor de transformación de ductilidad del S1GL equivalente 
(Bojórquez y Ruiz 2007) se obtiene $\mu_{I}=3.3$. Por lo tanto, considerando $v_{F O}=0.003$, y una ductilidad $\mu_{I}=3.3$, se obtiene un $C_{y r e q}=0.375$ a partir del espectro de la figura 11 para un periodo $T o=1.07 \mathrm{~s}$.

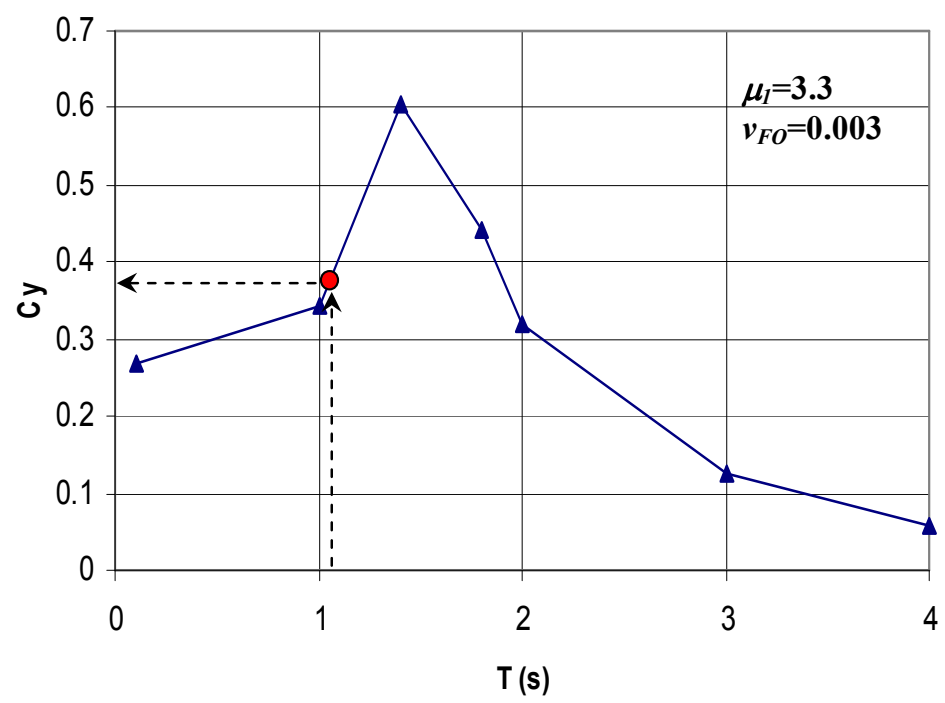

Figura 11. Espectro de ductilidad $\mu_{1}=3.3$ asociado a una tasa anual de falla de 0.003 . Comportamiento bilineal con $3 \%$ de rigidez de posfluencia y $\xi=3 \%$.

\section{Revisión de la resistencia lateral requerida $\left(C_{\text {yreal }} \geq C_{\text {yreq }}\right)$}

De un análisis de empuje lateral estático 'push-over' (figura 12) se obtiene $C_{y G}=0.41$. En la figura 12, el eje horizontal indica el desplazamiento máximo de azotea; mientras que el eje vertical representa el coeficiente sísmico, que es la relación entre el cortante basal dividido entre el peso total de la estructura. Dado que $C_{y G} \geq C_{y r e q}(0.41>0.375)$, entonces, se cumple la primera condición de diseño, y se continúa verificando la siguiente condición de aceptación.

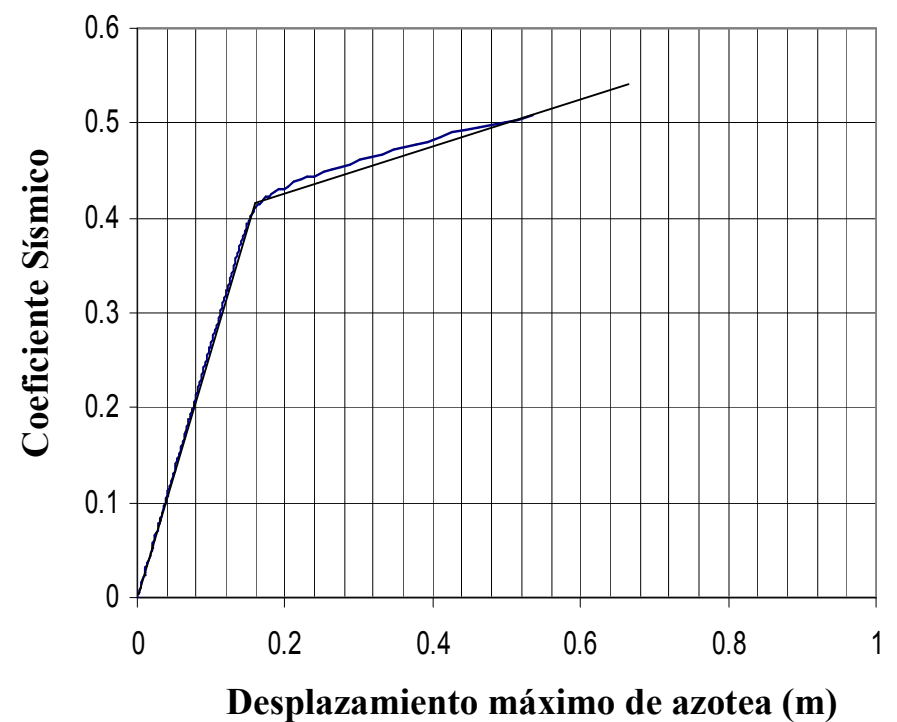

Figura 12. Análisis 'push-over' del marco estructural propuesto. 
4. Revisión de la distorsión máxima de entrepiso

Primero se determina la distorsión máxima del S1GL equivalente mediante la expresión 13, donde $\mu_{l}=3.3, H=28 \mathrm{~m}$. $D_{y l}$ que representa el desplazamiento de fluencia en el S1GL se obtiene con la expresión 14. En este caso $D_{y l}=0.117 \mathrm{~m}$.

Sustituyendo los valores se obtiene $\gamma_{1}=0.014$. Como se mencionó, la distorsión máxima de entrepiso en la estructura real se estima amplificando el valor de $\gamma_{l}$ mediante $T_{\gamma}$. Utilizando los resultados que se presentan en Bojórquez y Ruiz (2007), se obtiene para una $v(\gamma)=0.003$ un $T_{\gamma}=1.7$. De la ecuación 15 , se obtiene que $\gamma_{M}=0.023$ y debido a que $\gamma_{M}=0.023$ es igual a $\gamma_{t o}=0.023$, se satisface la ecuación 3.8 , y se concluye que la condición relativa a la distorsión máxima de entrepiso $\left(\gamma_{t o l} \geq \gamma_{M}\right)$ sí se cumple.

\section{Energía histerética normalizada que debe ser capaz de soportar la estructura}

La energía histerética requerida en el S1GL se obtiene mediante los espectros de energía histerética normalizada asociados a una tasa anual de falla de 0.003 . En la figura 13 se ilustra el espectro de energía histerética normalizada $E_{N}$, donde se obtiene mediante el periodo de la estructura $T o=1.07 \mathrm{~s}$ y el coeficiente sísmico real $C_{y G}=0.41$, una $E_{\text {NreqI }}=9$. Por lo tanto, con la ecuación 17 y el factor de transformación de energía histerética normalizada asociada a una $v\left(E_{N}\right)=0.003$ (Bojórquez y Ruiz 2007), se obtiene que la energía histerética normalizada requerida en la estructura es $E_{\text {NreqG }}=9 / 3.5=2.6$.

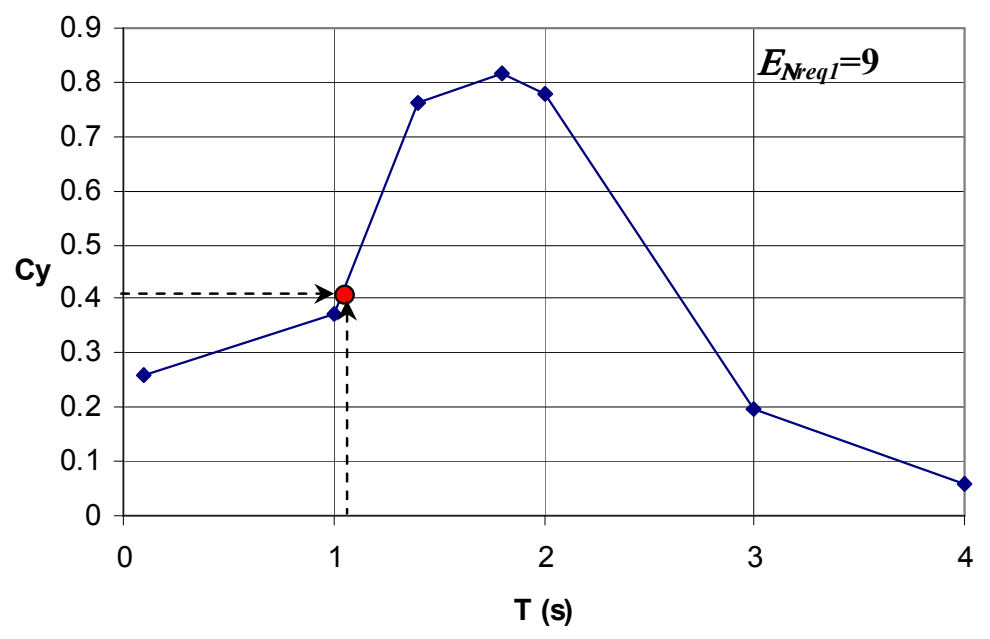

Figura 13. Espectro de energía histerética normalizada $\left(E_{N}\right)$ asociado a una tasa anual de falla de $0.003 \mathrm{y}$ una $E_{\text {HNreq }}=9$.

6. Revisión de la capacidad de disipación de energía 19):

Para satisfacer los requisitos de energía se requiere que se cumpla la siguiente condición (ecuación

$$
\frac{\sum_{i=1}^{N P}\left(2 N C Z_{f} F_{y} \theta_{p a} F p_{E H i}\right)}{C_{y G} D_{y G} W} \geq 2.6
$$


Para determinar el numerador del lado izquierdo de la ecuación 20 que corresponde a la capacidad total de disipación de energía histerética de la estructura se utiliza la tabla 2. En este ejemplo se supuso que $\theta_{p a}=0.05$ (Akbas et al. 2001, Choi y Shen, 2001), que para fines conservadores corresponde al límite inferior de capacidad de rotación obtenido en pruebas experimentales.

Tabla 2. Capacidad de disipación de energía histerética de la estructura.

\begin{tabular}{|c|c|c|c|c|c|c|}
\hline Nivel & $2 N_{C}$ & $f_{y}\left(\mathrm{~kg} / \mathrm{cm}^{2}\right)$ & $Z_{f}\left(\mathrm{~cm}^{3}\right)$ & $\theta_{p a}$ & $F_{E H}$ & $\Sigma($ kg.m) \\
\hline 1 & \multirow{9}{*}{6} & \multirow{9}{*}{2533} & 2328 & \multirow{9}{*}{0.05} & 0.20 & 3538 \\
\hline 2 & & & 3046 & & 0.94 & 21761 \\
\hline 3 & & & 3046 & & 0.98 & 22687 \\
\hline 4 & & & 3046 & & 0.69 & 15974 \\
\hline 5 & & & 2328 & & 0.42 & 7431 \\
\hline 6 & & & 1692 & & 0.24 & 3086 \\
\hline 7 & & & 1161 & & 0.14 & 1236 \\
\hline 8 & & & 1161 & & 0.08 & 706 \\
\hline Total & & & & & & 76419 \\
\hline
\end{tabular}

Haciendo todas las sustituciones en la ecuación 20 se obtiene:

$$
\frac{76419}{0.41(0.15)(663600)}=1.9<2.6
$$

Debido a que no se cumple la ecuación 20, el diseño no es satisfactorio, y la estructura debe rediseñarse. Es importante mencionar que al utilizar otro nivel de capacidad de rotación acumulada en los elementos, el diseño podría ser satisfactorio, por ejemplo para $\theta_{p a}=0.1$, lo cual indica que la condición impuesta de energía histerética normalizada, regirá o no, dependiendo de dicho parámetro; es decir, para estructuras con elementos de alta capacidad de rotación acumulada, un diseño convencional basado en resistencia y control de la distorsión máxima de entrepiso será adecuado. Es necesario que en un futuro se realicen más estudios para poder evaluar de una manera más real la capacidad de rotación plástica acumulada en elementos. El procedimiento aquí propuesto puede utilizarse usando los valores de la rotación plástica acumulada en los elementos que resultan de análisis experimentales futuros.

Debido a que el diseño no fue satisfactorio, se procede a rediseñar la estructura, incrementando ligeramente las secciones, y se repite el procedimiento a partir del tercer paso. El nuevo diseño se ilustra en la tabla 3 y se regresa al paso 2 .

Tabla 3. Secciones propuestas para la estructura en la segunda iteración.

\begin{tabular}{|c|c|c|c|}
\hline Nivel & Vigas & $\begin{array}{c}\text { Columnas } \\
\text { Internas }\end{array}$ & $\begin{array}{c}\text { Columnas } \\
\text { Externas }\end{array}$ \\
\hline 1 & W24x94 & W36x194 & W36x194 \\
\hline 2 & W24x94 & W36x194 & W36x194 \\
\hline 3 & W24x94 & W36x170 & W36x170 \\
\hline 4 & W24x94 & W36x170 & W36x170 \\
\hline 5 & W24x84 & W36x150 & W36x150 \\
\hline 6 & W21x 73 & W36x150 & W36x150 \\
\hline 7 & W21x57 & W36x135 & W36x135 \\
\hline 8 & W $21 \times 50$ & W36x135 & W36x135 \\
\hline
\end{tabular}




\section{SEGUNDA ITERACIÓN}

2. Resistencia lateral requerida para el control de la ductilidad máxima

En este caso el periodo resulta $T o=1.04 \mathrm{~s}$. De la figura 14 se obtiene un coeficiente sísmico requerido $C_{\text {yreq }}=0.36$.

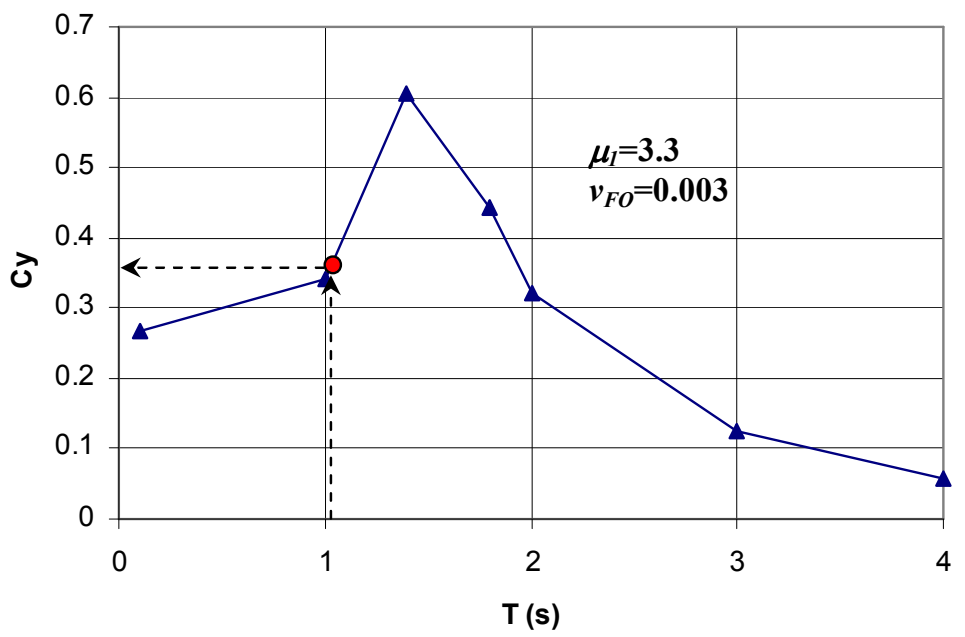

Figura 14. Espectro de ductilidad $\mu_{I}=3.3$ asociado a una tasa anual de falla de 0.003 .

3. Revisión de la Resistencia Lateral Requerida $\left(C_{y r e a l} \geq C_{y r e q}\right)$

De un análisis 'push-over' (figura 15) se obtiene $C_{\text {yreal }}=0.43$. Como $C_{\text {yreal }} \geq C_{\text {yreq }}(0.43>0.36)$, entonces se cumple la primera condición de diseño, y se continúa verificando las siguientes condiciones de aceptación.

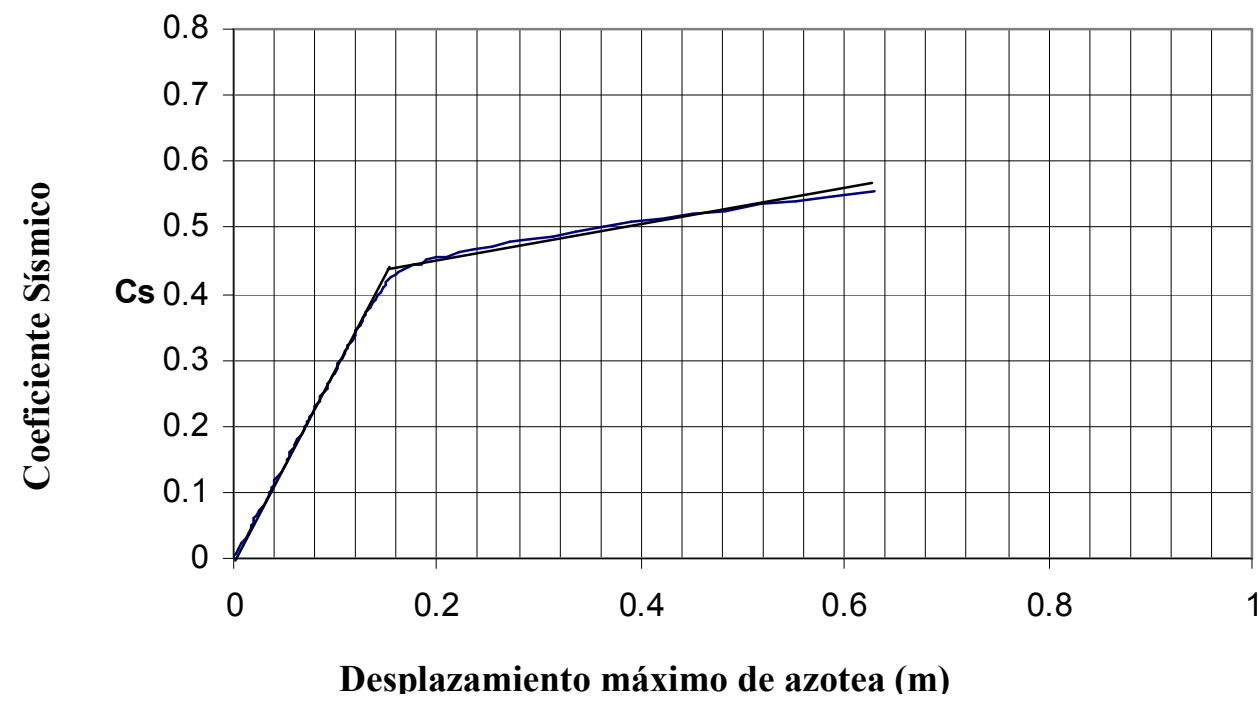

Figura 15. Análisis ‘push-over’ del marco estructural propuesto (segunda iteración). 
4. Revisión de la distorsión máxima de entrepiso

Para esta estructura, $\mu_{I}=3.3, H=28 \mathrm{~m}$ y $D_{y l}$ que representa el desplazamiento de fluencia en el S1GL se obtiene con la expresión 14.

Sustituyendo los valores se obtiene $\gamma_{I}=0.0136$ y la distorsión máxima de entrepiso resulta $\gamma_{M}=0.023$. Debido a que $\gamma_{M}=0.023$ es igual a $\gamma_{t o}=0.023$, se satisface la ecuación 16 , y por lo tanto se cumple está condición de aceptación del diseño.

\section{Energía histerética normalizada que debe ser capaz de soportar la estructura}

En la figura 16 se ilustra el espectro de $E_{N}$, para el periodo de la estructura $T o=1.04 \mathrm{~s}$ y el coeficiente sísmico real $C_{\text {yreal }}=0.43$ se obtiene $E_{\text {NreqI }}=7$. Por lo tanto, se tiene que la energía histerética normalizada requerida en la estructura es $E_{\text {NreqG }}=7 / 3.5=2$.

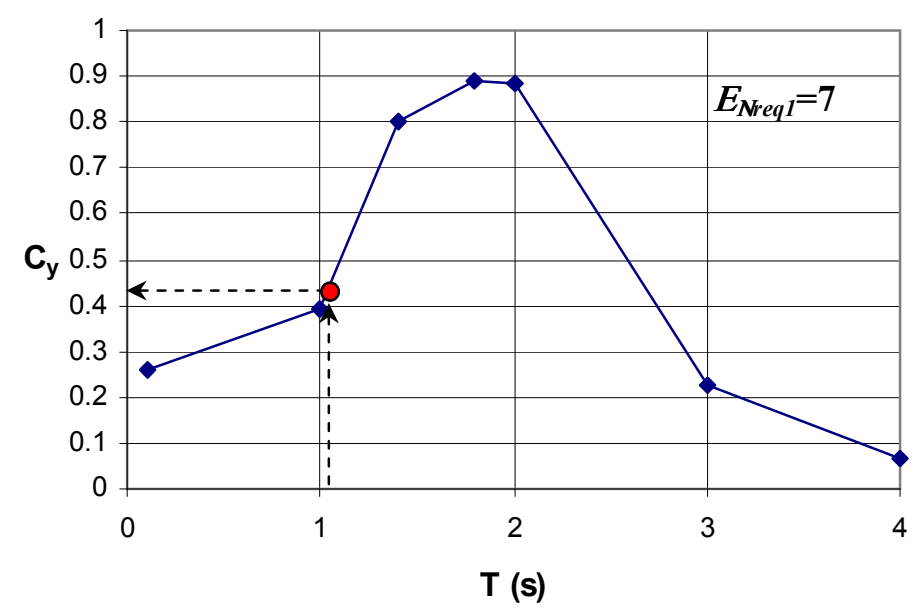

Figura 16. Espectro de energía histerética normalizada asociado a una tasa anual de falla de 0.003 y una

$$
E_{\text {Nreq1 }}=7 \text {. }
$$

6. Revisión de la capacidad de disipación de energía

Se revisa la ecuación 19 con ayuda de la tabla 4:

$$
\frac{\sum_{i=1}^{N P}\left(2 N C Z_{f} F_{y} \theta_{p a} F p_{E H i}\right)}{C_{y G} D_{y G} W} \geq 2
$$

Haciendo las sustituciones en la ecuación anterior, se obtiene:

$$
\frac{79511}{0.43(0.14)(663600)}=2 \geq 2
$$

La estructura propuesta cumple con el balance de energía y la tercera y última condición de diseño, por lo tanto el diseño final propuesto es adecuado. 
Tabla 4. Capacidad de disipación de energía histerética de la estructura.

\begin{tabular}{ccccccc}
\hline Nivel & $\mathbf{2 N}_{\boldsymbol{C}}$ & $\boldsymbol{f}_{\boldsymbol{v}}\left(\mathbf{k g} / \mathbf{c m}^{2}\right)$ & $\boldsymbol{Z}_{f}\left(\mathbf{c m}^{3}\right)$ & $\boldsymbol{\theta}_{\boldsymbol{p a}}$ & $\boldsymbol{F}_{E H}$ & $\boldsymbol{\Sigma}(\mathbf{k g . m})$ \\
\hline 1 & & & 3046 & & 0.20 & $\mathbf{4 6 3 0}$ \\
2 & & & 3046 & & 0.94 & $\mathbf{2 1 7 6 1}$ \\
3 & & & 3046 & & 0.98 & $\mathbf{2 2 6 8 7}$ \\
4 & \multirow{2}{*}{2533} & 3046 & 0.05 & 0.69 & $\mathbf{1 5 9 7 4}$ \\
5 & & & 2655 & & 0.42 & $\mathbf{8 4 7 6}$ \\
6 & & & 2062 & & 0.24 & $\mathbf{3 7 6 1}$ \\
7 & & & 1425 & & 0.14 & $\mathbf{1 5 1 6}$ \\
8 & & & 1161 & & 0.08 & $\mathbf{7 0 6}$ \\
Total & & & & & & $\mathbf{7 9 5 1 1}$
\end{tabular}

\section{DESEMPEÑO SÍSMICO DEL MARCO ESTRUCTURAL}

En esta parte se presenta el desempeño sísmico del marco estructural diseñado con el procedimiento propuesto, así como de un marco con la misma configuración estructural pero diseñado de acuerdo con el RCDF-2004. El marco obtenido con el Reglamento, fue diseñado usando parámetros que son consistentes con aquellos utilizados en el ejemplo ilustrativo; particularmente: a) se utilizó un factor de comportamiento sísmico $\mathrm{Q}=3$; y b) espectros correspondientes a la zona IIIb del valle de México. Una información detallada acerca de este diseño se encuentra en (Díaz, 2006). Las secciones finales de ambos diseños se ilustran en las tablas 5 y 6 .

Tabla 5. Secciones finales obtenidas con el criterio de diseño propuesto $(T o=1.04 \mathrm{~s})$.

\begin{tabular}{|c|c|c|c|}
\hline Nivel & Vigas & $\begin{array}{c}\text { Columnas } \\
\text { Internas }\end{array}$ & $\begin{array}{c}\text { Columnas } \\
\text { Externas }\end{array}$ \\
\hline 1 & W24x94 & W36x194 & W36x194 \\
\hline 2 & W24x94 & W36x194 & W36x194 \\
\hline 3 & W24x94 & W36x170 & W36x170 \\
\hline 4 & W24x94 & W36x170 & W36x170 \\
\hline 5 & W24x84 & W36x150 & W36x150 \\
\hline 6 & W21x73 & W36x150 & W36x150 \\
\hline 7 & W21x57 & W36x135 & W36x135 \\
\hline 8 & W21 150 & W36x135 & W36x135 \\
\hline
\end{tabular}

Tabla 6. Secciones obtenidas con el RCDF-2004 $(T o=1.20 \mathrm{~s})$.

\begin{tabular}{|c|c|c|c|}
\hline Nivel & Vigas & $\begin{array}{c}\text { Columnas } \\
\text { Internas }\end{array}$ & $\begin{array}{c}\text { Columnas } \\
\text { Externas }\end{array}$ \\
\hline 1 & W21x83 & W36x210 & W36x194 \\
\hline 2 & W21x93 & W36x210 & W36x194 \\
\hline 3 & W21x93 & W36x194 & W36x182 \\
\hline 4 & W21x83 & W36x194 & W36x182 \\
\hline 5 & W18x71 & W36x170 & W36x160 \\
\hline 6 & W18x65 & W36x170 & W36x160 \\
\hline 7 & W18x55 & W36x160 & W36x135 \\
\hline 8 & W18x46 & W36x160 & W36x135 \\
\hline
\end{tabular}


Ambas estructuras se sujetaron al registro sísmico obtenido en suelo blando del valle de México durante el sismo del 19 de septiembre de 1985. El registro fue escalado para un periodo de retorno de $0.003^{-1}$ años según el periodo de vibración de cada estructura.

En la figura 16 se comparan las distorsiones de entrepiso. Se observan diferencias entre ambos diseños. Las distorsiones son mayores en la estructura diseñada con el RCFD-2004. Por ejemplo, la distorsión máxima de entrepiso en este marco es de alrededor de 0.026, mientras que en el marco diseñado con el procedimiento planteado es de 0.016 . Esto representa una reducción del $38 \%$ en las distorsiones cuando se utiliza el criterio aquí planteado. La distorsión de entrepiso es el principal parámetro usado para diseño sísmico en la mayoría de los códigos del mundo. Se concluye que el procedimiento propuesto influye en el nivel de confiabilidad estructural a través de dar lugar a una estructura que exhibe importantes reducciones en los niveles de desplazamientos máximos de entrepiso. Note que esto se logra con un ligero incremento (del 3\%) en el peso de la estructura.

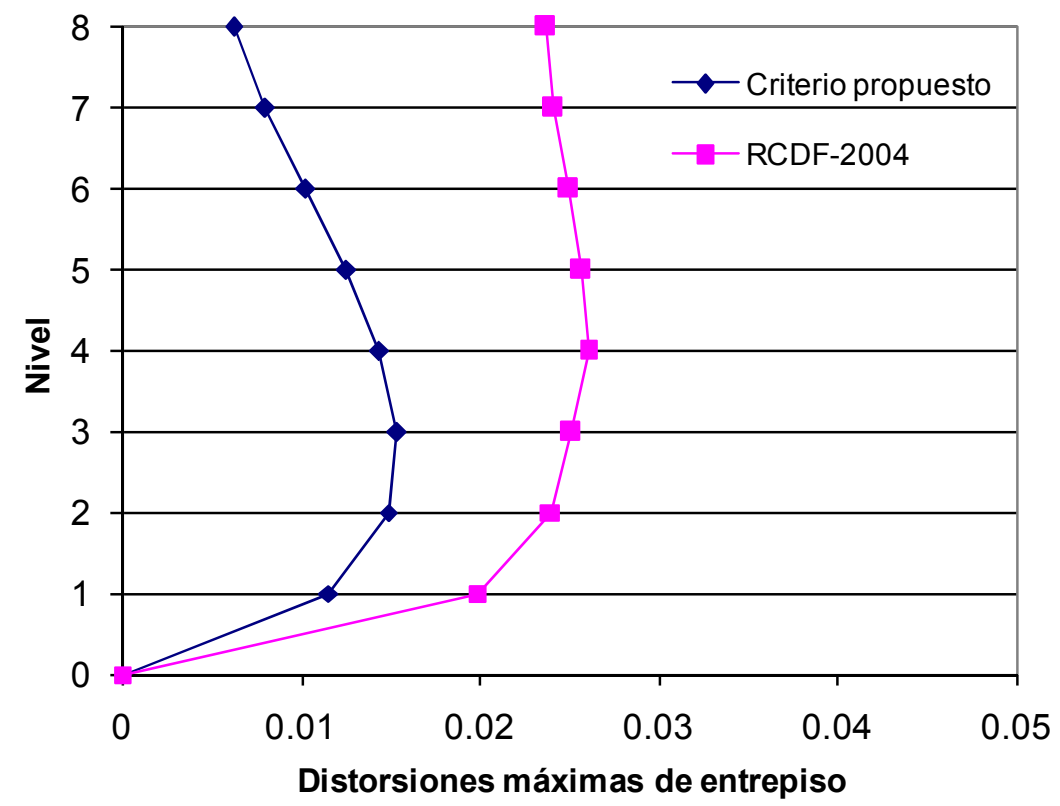

Figura 16. Distorsiones de entrepiso en los marcos estructurales sujetos al sismo de 1985 registrado en la estación SCT escalado para un periodo de retorno de $0.003^{-1}$ años.

\section{CONCLUSIONES}

Se propuso un procedimiento de diseño sísmico de marcos estructurales de acero basado en confiabilidad estructural que considera de manera explícita la demanda de energía histerética. El procedimiento toma en cuenta la confiabilidad y las demandas acumuladas en las estructuras a través de espectros de energía histerética normalizada con TAFU. El criterio se aplicó para diseñar una estructura de acero de ocho niveles. Su desempeño sísmico se compara con el de un marco estructural diseñado de acuerdo con el RCDF- 2004. Se observa un mejor desempeño estructural en el diseño obtenido con el criterio aquí planteado, con una reducción del $38 \%$ en la distorsión máxima de entrepiso.

Se hace ver que las demandas de deformación plástica tienen importancia significativa para estructuras con elementos de baja capacidad de rotación plástica acumulada sujetas a movimientos de larga duración. En el caso de estructuras con elementos de alta capacidad de rotación plástica acumulada, 
un diseño sísmico basado en resistencia y en el control de las distorsiones máximas de entrepiso resulta aceptable siempre y cuando no se sometan a demandas sísmicas severas. En conclusión, existen dos casos en los que es de importancia considerar las demandas acumuladas por deformaciones plásticas. La primera corresponde a estructuras con baja capacidad de acomodar deformaciones plásticas y la segunda a estructuras sujetas a demandas sísmicas severas, tal es el caso de estructuras ubicadas en terreno blando del valle de México.

El complemento de las metodologías actuales de diseño sísmico con el criterio de diseño sísmico basado en confiabilidad propuesto aquí puede dar lugar a mejores diseños de estructuras sujetas a la acción de movimientos sísmicos de larga duración.

La manera en que está planteado el criterio aquí propuesto es simple con la finalidad de que pueda aplicarse en la práctica de la ingeniería sísmica. Sin embargo, se debe resaltar que son necesarios mayores estudios para confirmar las hipótesis aquí expuestas. Por ejemplo, se debe contar con suficiente información experimental para validar y reducir la incertidumbre al suponer una capacidad de rotación plástica acumulada en elementos de acero. Se debe proporcionar a los ingenieros de la práctica los espectros de diseño con TAFU, para ductilidad y energía histerética normalizada. De preferencia se debe contar con un software donde puedan determinarse dichos espectros para las distintas zonas del valle de México. Finalmente, a medida que se cuente con información experimental que permita reducir la incertidumbre que existe para evaluar la capacidad estructural, medida en términos de cualquier parámetro, la aplicabilidad de métodos de diseño sísmico basados en energía y confiabilidad estructural estará más al alcance de la práctica de la ingeniería.

\section{AGRADECIMIENTOS}

Se agradecen los comentarios y orientación de la Dra. Sonia E. Ruiz Gómez y del Dr. Amador Terán Gilmore, con quienes tuve discusiones de gran valía que enriquecieron el trabajo aquí presentado. El autor también agradece a CONACYT la beca otorgada durante los estudios de posgrado y dentro del programa de Repatriación. Este trabajo se realizó con el apoyo de la Universidad Autónoma de Sinaloa dentro del proyecto PROFAPI 2009/026 y de la UNAM dentro del proyecto DGAPA-IN108708.

\section{REFERENCIAS}

AISC (2003), "LRFD design specification for structural steel buildings", American Institute of Steel Construction, Chicago, IL.

Akbas, B, J Shen y H Hao (2001), "Energy approach in performance-based design of steel moment resisting frames for basic safety objective", The Structural Design of Tall Buildings, Vol. 10, pp. 193-217.

Akiyama, H (1985), "Earthquake-Resistant Limit-State Design for Buildings", University of Tokyo Press, Tokyo.

Arroyo, D y M Ordaz (2007), "Hysteretic energy demands for SDOF systems subjected to narrow band earthquake ground motions. Applications to the lake bed zone of Mexico City", Journal of Earthquake Engineering, Vol. 11, pp. 147-165.

Baker, J W y C A Cornell (2005), "A vector-valued ground motion intensity measure consisting of spectral acceleration and epsilon", Earthquake Engineering and Structural Dynamics, Vol. 34, pp. 1193-1217. 
Baker, J W y C A Cornell (2008), "Vector-valued intensity measures for pulse-like near-fault ground motions", Engineering Structures, Vol. 30(4), pp. 1048-1057.

Bazzurro, P (1998), "Probabilistic seismic demand analysis", Ph.D. Thesis, Stanford University.

Bertero, R D y V V Bertero (1992), "Tall reinforced concrete buildings: conceptual earthquake-resistant design methodology", Report No.UCB/EERC-92/16, University of California.

Bojórquez, E y S E Ruiz (2004), "Strength reduction factors for the valley of Mexico taking into account low cycle fatigue effects", $13^{\circ}$ World Conference on Earthquake Engineering, paper 516, Vancouver, Canada 2004 (CD-ROM).

Bojórquez, E, S E Ruiz y L Esteva (2005), "Funciones de transformación de respuestas máximas entre marcos estructurales y sus correspondientes S1GL con probabilidades de falla similares", $X V$ Congreso Nacional de Ingeniería Sísmica (CD), Ciudad de México 2005.

Bojórquez, E, M A Díaz, S E Ruiz, A Terán-Gilmore (2006), "Correlation between local and global cyclic structural capacity of SMR frames", First European Conference on Earthquake Engineering and Seismology (CD), Geneva Switzerland.

Bojórquez, E, y S E Ruiz (2007), "Factores de transformación de ductilidades, distorsiones máximas de entrepiso y de energía histerética normalizada entre S1GL y SMGL", Tercer Congreso Nacional de Ingeniería Sísmica de España, Girona, España.

Bojórquez, E (2007), "Vulnerabilidad sísmica de edificios usando conceptos de energía", Tesis de Doctorado, Universidad Nacional Autónoma de México.

Bojórquez, E, M A Díaz, S E Ruiz, F García-Jarque (2007), “Confiabilidad sísmica de varios edificios (cuatro a diez niveles) localizados en suelo blando de la ciudad de México, diseñados con el RCDF2004”, Revista de Ingeniería Sísmica, Vol. 76, pp. 1-27.

Bojórquez, E y I Iervolino (2009), "Spectral shape proxies and nonlinear structural response". Artículo en revisión.

Bojórquez E, A Reyes-Salazar, A Terán-Gilmore A y S E Ruiz (2009), "Energy-based damage index for steel structures". Artículo en revisión.

Chan, S, S E Ruiz y M A Montiel (2005), "Escalamiento de acelerogramas y número mínimo de registros requeridos para el análisis de estructuras”, Revista de Ingeniería Sísmica, Vol. 72, pp. 1-24.

Choi, B y J Shen (2001), “The establishing of performance level thresholds for steel moment-resisting frames", The Structural Design of Tall Buildings, Vol. 10, pp. 193-217.

Choi, H y J Kim (2006), "Energy-based seismic design of buckling-restrained braced frames using hysteretic energy spectrum", Engineering Structures, Vol. 28, pp. 304-311.

Cornell, C A (1968), "Engineering seismic risk analysis", Bulletin of the Seismological Society of America, Vol. 58, $\mathrm{N}^{\mathrm{0}}$ 5, pp. 1583-1606.

Cornell C A (1992), "Reliability-based earthquake-resistant design: the future", Proceedings, 11th World Conference on Earthquake Engineering, Balkema Rotterdam 1992, Paper No. 2166.

Díaz, M A (2006), “Confiabilidad sísmica de edificios de acero diseñados con el RCDF-2004”, Tesis presentada en la DEPFI para obtener el grado de Maestro en Ingeniería, UNAM.

Esteva, L (1967), “Criterios para la construcción de espectros para diseño por sismo”, Boletín del Instituto de Materiales y Modelos Estructurales, 19, Universidad Central de Venezuela. 
Esteva, L, S E Ruiz y J L Rivera (2005), "Reliability and performance-based design of structures with energy-dissipating devices", 9th World Seminar on seismic isolation, energy dissipation and active vibration control of structures, Kobe, Japón.

Fajfar, P y H Krawinkler (1997), "Conclusions and recommendations”, Seismic Design Methodologies for the Next Generation of Codes, A. A. Balkema.

Ghosh, S y K Collins (2002), "Application of uniform hazard energy spectra in reliability-based seismic design", 7th U.S. National Conference on Earthquake Engineering.

Huerta, B y E Reinoso (2002), "Espectros de energía de movimientos fuertes registrados en México", Revista de Ingeniería Sísmica, SMIS, Vol. 66, pp. 45-72.

Housner, G W (1956), "Limit design of structures to resist earthquakes", First World Conference on Earthquake Engineering, Berkeley, California 1956.

Inoue, T y Cornell, C A (1991), "Seismic hazard analysis of MDOF structures", ICASP 6, Ciudad de México, Vol. 1, pp. 437-444.

Krawinkler, H y A Nassar (1992), "Seismic design based on ductility and cumulative damage demands and capacities", Nonlinear Seismic Analysis and Design of Reinforced Concrete Buildings (Editors: H. Krawinkler and P. Fajfar), Elsevier Applied Science, U.K., pp. 95-104.

Moehle J P (1992), "Displacement based design of reinforced concrete structures subjected to earthquakes", Earthquake Spectra, Vol. 8, № 3, pp. 403-428.

Park, Y J y A H Ang (1985), "Mechanistic seismic damage model for reinforced concrete", ASCE Journal of Structural Engineering, Vol. 111, No. ST4, pp. 740-757.

Popov, E, K C Tsai y M Engelhardt (1989), “On seismic steel joints and connections", Engineering Structures, Vol. 11, pp. 148-162.

Priestley, M J N (2000), "Performance based seismic design", 12 World Conference on Earthquake Engineering, Paper 2831.

Reglamento de Construcciones del Distrito Federal RCDF (2004).

Rivera J L (2006), "Espectros de confiabilidad uniforme para sistemas estructurales con disipadores de energía", Tesis de Doctorado, Universidad Nacional Autónoma de México.

Rivera, J L y S E Ruiz (2007), "Design approach based on UAFR spectra for structures with displacement-dependent dissipating elements", Earthquake Spectra Vol. 23, pp. 417-439.

Rodríguez, M E y J C Ariztizabal (1999), "Evaluation of a seismic damage parameter", Earthquake Engineering and Structural Dynamics, Vol. 28, pp. 463-477.

Shome, N y C A Cornell (1999), "Probabilistic seismic demand analysis of nonlinear structures", Reliability of Marine Structures Program 1999, Report No. RMS-35, Dept. of Civil Eng., Stanford University.

Shome, N (1999), "Probabilistic seismic demand analysis of nonlinear structures", Ph.D. Thesis, Stanford University.

Silva-Olivera, H y O López-Bátiz (2001), "Estudio experimental sobre índices de daño en estructuras de concreto reforzado sujetas a cargas laterales", XIII Congreso Nacional de Ingeniería Sísmica, Guadalajara, México (CD_ROM).

Stephens, J E y J T P Yao (1987), "Damage assessment using response measurements", ASCE Journal of Structural Engineering, Vol. 113, No. 4, 787-801. 
Terán-Gilmore, A (1996), "Performance-based earthquake-resistant design of framed building using energy concepts", Tesis de Doctorado, University of California Berkley.

Terán-Gilmore, A (2001), "Consideraciones del uso de la energía plástica en el diseño sísmico", Revista de ingeniería Sísmica, SMIS, Vol. 65, pp. 81-110.

Terán-Gilmore, A y J O Jirsa (2005), “A damage model for practical seismic design that accounts for low cycle fatigue", Earthquake Spectra, Vol. 21, No. 3, pp. 803-832.

Teran-Gilmore, A y R Simon (2006), "Use of constant cumulative ductility spectra for performance-based seismic design of ductile frames", 8th U.S. National Conference on Earthquake Engineering.

Tothong, P y N Luco (2007), "Probabilistic seismic demand analysis using advanced ground motion intensity measures", Earthquake Engineering and Structural Dynamics Vol. 36, pp. 1837-1860.

Uang C M y V V Bertero (1990), "Evaluation of seismic energy in structures", Earthquake Engineering and Structural Dynamics, Vol. 19, pp. 77-90.

Wen, Y K (1995). "Building reliability and code calibration”, Earthquake Spectra, Vol. 11, N 2, pp 269296.

Williams, M S y R G Sexsmith (1997), "Seismic assessment of concrete bridges using inelastic damage analysis", Engineering Structures, Vol. 19, No. 3, pp. 208-216. 